\title{
Explorando o Uso da Robótica na Educação Básica: um estudo sobre ações práticas que estimulam o Pensamento Computacional
}

\author{
Isabelle M. L. Souza ${ }^{1}$, Lívia M. R. Sampaio ${ }^{1}$, Wilkerson L. Andrade ${ }^{1}$ \\ ${ }^{1}$ Universidade Federal de Campina Grande (UFCG), Campina Grande, Brasil \\ isabellemarialcopin.ufcg.edu.br, \{wilkerson, livia\}@computacao.ufcg.edu.br
}

\begin{abstract}
Robotics laboratories for Basic Education schools (BE) are often acquired aiming teaching sciences. However, the use of robotics in EB is difficult due to limitations of physical structure, academic materials, and training of professionals. Thus, we carried a work, from the organization of the laboratories to the offer of classes, with the purpose of facilitating the use of robotics in state schools. We analyzed the effects of the mentioned work considering the vision of the school managers and the evolution of the Computational Thinking (CT) of 41 high school freshman. The results indicate that the use of robotics to BE considering physical and methodological factors motivates teachers and students, stimulates students' learning and CT skills.
\end{abstract}

Resumo. Laboratórios de robótica para Educação Básica(EB) frequentemente são adquiridos visando o ensino das ciências. Contudo, o uso da robótica na EB é dificultado por limitações de estrutura física, materiais didáticos e formação de profissionais. Assim, com o intuito de facilitar o uso da robótica em escolas estaduais de EB, realizamos um trabalho desde a organização dos laboratórios, até a oferta de aulas. Analisamos os efeitos do trabalho considerando a visão dos gestores escolares e a evolução do Pensamento Computacional (PC) de 41 alunos de $1^{a}$ Série do Ensino Médio. Os resultados indicam que o uso da robótica na EB considerando fatores físicos e metodológicos motiva professores e alunos, estimula o aprendizado e as habilidades do PC dos alunos.

\section{Introdução}

O Pensamento Computacional (PC), segundo [Wing 2006], envolve habilidades relacionadas à resolução de problemas que podem ser estimuladas quando conceitos da Ciência da Computação (CC) são trabalhados. O PC pode ser aplicado em diferentes contextos, aumentando a produtividade, criatividade e raciocínio lógico, sendo tão importante quanto à capacidade de ler e escrever. Em particular, aplicações do PC na Educação Básica (EB) contribuem para o desenvolvimento cognitivo dos alunos.

Segundo o [Dicionário Interativo da Educação Brasileira 2015], a Robótica Educacional (RE) envolve ambientes compostos por materiais físicos montáveis e controláveis por computadores. Esses ambientes apoiam a educação possibilitando o desenvolvimento de habilidades, como: trabalho em equipe, raciocínio lógico e criatividade. Assim, é possível vislumbrar na RE possibilidades para estímular o PC nos níveis de ensino, especialmente na EB. Pesquisas, como a do entusiasta da robótica na educação 
VII Congresso Brasileiro de Informática na Educação (CBIE 2018)

Anais dos Workshops do VII Congresso Brasileiro de Informática na Educação (WCBIE 2018)

[Papert 1980], indicam que a RE é capaz de contribuir com o ensino das ciências, assim como de programação. No Brasil, instituições de EB investem em RE buscando o desenvolvimento acadêmico de alunos aliando teoria e prática. O governo do estado da Paraíba implantou, em escolas de Ensino Médio (EM), laboratórios de robótica compostos pela metodologia Brink Mobil ${ }^{\circledR}$ com a proposta de envolver professores e alunos em atividades de diversas áreas do conhecimento. No entanto, a utilização desses enfrenta obstáculos que, por vezes, impossibilita a utilização da robótica conforme esperado.

Nesse âmbito, este trabalho busca identificar o impacto que o uso da robótica causa na EB, sob a perspectiva da organização do espaço físico do laboratório, a oferta de aulas com as ferramentas disponíveis no laboratório e o aprendizado em computação pelos alunos. Para isso, realizamos um estudo em escolas do estado da Paraíba buscando responder às seguintes perguntas: Q1: Como a inserção da robótica impacta escolas estaduais de EB? Q2: Como a estrutura física e organizacional dos laboratórios influenciam na efetivação do ensino com robótica em escolas de EB? Q3: Como o ensino com robótica estimula as habilidade do Pensamento Computacional de alunos da EB?

Realizamos um estudo qualitativo seguindo o método de pesquisa ação, dividido em três fases: 1) Seleção e organização de laboratórios de robótica de escolas estaduais de EB; 2) Construção e aplicação de atividades com robótica para alunos da $1^{\text {a }}$ Série do EM; 3) Exploração dos efeitos das intervenções com robótica sobre os participantes.

Os resultados obtidos indicaram que o uso da robótica metodologicamente elaborado é capaz de impactar positivamente a formação e sobretudo a motivação de alunos e professores da EB. Foi evidenciado que a organização física dos laboratórios de robótica é avaliada por gestores e alunos como um fator importante e, que aulas de robótica realizadas em estrutura física organizada podem motivar o aprendizado, assim como professores que passam a utilizar a ferramenta no ensino quando condições mínimas são disponibilizadas. Por fim, verificamos que $80 \%$ dos alunos da escola A e $38 \%$ da escola B apresentaram melhora no desempenho dos testes relacionados ao PC. Os dados indicam que o desempenho em PC de alunos da EB pode ser melhorado quando a robótica é utilizada no ensino considerando fatores físicos, estruturais e metodológicos.

Este trabalho segue organizado nas seguintes seções: a Seção 2 apresenta o contexto envolvendo conceitos sobre PC, Robótica Educacional e o Projeto BrinkRobótica implantado em escolas estaduais de EB; a Seção 3 descreve os passos metodológicos; a Seção 4 apresenta os resultados, discussões e ameaças à validade da pesquisa e, por fim, a Seção 5 expõe as principais conclusões e trabalhos futuros.

\section{Contextualização do Trabalho}

Essa seção apresenta conceitos e práticas consolidadas na literatura, relacionadas com PC e RE, aspectos importantes para compreensão do contexto e problema de pesquisa.

\subsection{Pensamento Computacional}

O PC apoia-se na forma como cientistas da computação buscam soluções para problemas. [França et al. 2012] consideram que o PC favorece habilidades cognitivas, impulsiona a eficiência, senso crítico e criatividade, pois estimula a capacidade de resolver problemas.

[Wing 2006] defende que PC "é uma habilidade fundamental para todos, não somente para cientistas da computação". Estudos recentes, como apresentado por 
VII Congresso Brasileiro de Informática na Educação (CBIE 2018)

Anais dos Workshops do VII Congresso Brasileiro de Informática na Educação (WCBIE 2018)

[Araujo et al. 2016], reforçam as afirmações de [Papert 1980], dando significado e ampliando as discussões do impacto que o PC causa nas áreas do saber. Os conceitos da CC abrangem habilidades mentais para concepção e resolução de problemas observadas em procedimentos típicos de cientistas computação, tais como quando decompõem, abstraem, usam recursividade e esquematizam algoritmos para resolver problemas. Conceitos, que Wing considera tão significantes quanto a capacidade de ler, escrever e contar, sendo indicado inseri-los no trabalho das habilidades cognitivas das crianças.

\subsection{Robótica Educacional}

Robótica é uma ciência que envolve vários conceitos, como computação, engenharia, física, neurociência e inteligência artificial, com o objetivo de criar e se utilizar de robôs [Halfpap et al. 2005]. A RE firmou-se com o início das demandas por práticas e ferramentas para o ensino e aprendizagem. Esse fato resultou na inserção da robótica no ambiente escolar como ferramenta pedagógica, conforme difundido por [Santos et al. 2016].

O termo RE refere-se a espaços escolares com materiais (sucata ou kits) destinados a manipulação de peças, sensores e motores controláveis. Estudos indicam que a RE facilita o desenvolvimento de habilidades, como trabalho em equipe, raciocínio lógico e criatividade. [Gerber et al. 2017] indica a RE para o ensino de ciências, pois permite a inclusão e consolidação do conhecimento de maneira lúdica na EB. Para [Strnad 2017] o uso da RE em competições estimula a aprendizagem, facilita o pensamento lógico, criatividade e aumenta o interesse das crianças da EB em aprender programação.

\subsection{Pensamento Computacional na Educação Básica}

As competências do PC podem ser desenvolvidas na EB de diferentes formas, integradas ou não ao currículo escolar. Para [Matos 2013] é preciso envolver o aprendiz em diversas situações problema que aliem o ensino das ciências às necessidade educacionais. A [Computer Science Teachers Association 2011] ao delinear o Model Curriculum for K-12 Computer Science, menciona os nove fundamentos do PC de [Barr and Stephenson 2011] e recomenda o ensino de computação em escolas.

Os autores de [Rodrigues et al. 2015] analisam os efeitos do PC no desempenho de alunos da EB e evidenciam uma correlação entre as habilidades em programação e o desempenho de alunos no ENEM. [Costa et al. 2016] verificaram nos $8^{\circ}$ e $9^{\circ}$ ano da EB que questões matemáticas alinhadas ao PC estimulam a resolução de problemas. [Souza et al. 2016] indicam que o ensino de robótica com ênfase em PC impacta positivamente o desempenho de alunos do EM.

Dessa forma, é inegável o potencial que o PC pode oferecer à formação de alunos da EB. No que tange sua forma de ensino, verifica-se uma pluralidade de ações alinhadas com as ideias levantadas por [Wing 2006].

\subsection{Projeto Brink Robótica nas Escolas Estaduais da Paraíba}

As escolas do Estado da Paraíba passaram a receber, em 2012, laboratórios da Brink Mobil $^{\circledR}$ intitulado de Brink Robótica. O projeto visa o ensino de ciências relacionando a teoria com a prática através da metodologia Brink Mobil ${ }^{\circledR}$ e os materiais da

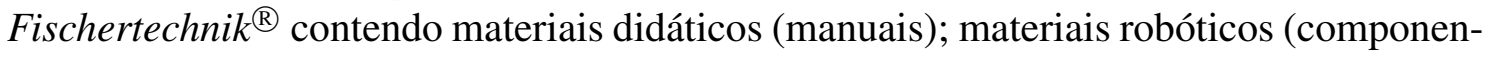
tes eletrônicos, peças de montagens e softwares); além de uma proposta metodológica. 
VII Congresso Brasileiro de Informática na Educação (CBIE 2018)

Anais dos Workshops do VII Congresso Brasileiro de Informática na Educação (WCBIE 2018)

A Brink Robótica busca garantir práticas que envolvem professores e alunos em diversas áreas do conhecimento. Não obstante, a concretização da proposta do Governo da Paraíba enfrenta adversidades, que por vezes impossibilita a efetivação da robótica. Embora as escolas tenham recebido materiais, evidências indicam que o uso da robótica é influenciado pela estrutura física, limitações de materiais didáticos, manutenção dos equipamentos, formação de profissionais; fatores que impactam a motivação e aprendizado de alunos e professores no contexto do projeto Brink Robótica na Paraíba.

\section{Metodologia}

Este estudo é qualitativo e prático. É qualitativo por buscar entender a interpretação que os envolvidos atribuíram às condutas e experiências com RE. É uma pesquisa ação por perceber uma dificuldade real, ter apoio de professores, apresentar soluções, executar intervenções e julgar resultados [Cohen et al. 2002]. O estudo foi dividido em três fases: 1) Seleção e organização de laboratórios de robótica de escolas estaduais de EB; 2) Construção e aplicação de atividades com robótica para alunos da $1^{\text {a }}$ Série do EM; 3) Exploração dos efeitos das intervenções com robótica sobre os alunos participantes.

Sobretudo, este estudo busca identificar o impacto que o uso da robótica causa na EB, sob a perspectiva da organização do espaço físico do laboratório realizado nas escolas. Buscamos responder as seguintes questões: Q1: Como a inserção da robótica impacta escolas de EB? Q2: Como a estrutura física e organizacional dos laboratórios influenciam na efetivação do ensino com robótica em escolas de EB? Q3: Como o ensino com robótica influencia as habilidades do Pensamento Computacional de alunos da EB?

\subsection{Seleção das Escolas e Organização dos Laboratórios}

Foram selecionadas duas escolas estaduais (A e B) localizadas na zona sul da cidade de Campina Grande na Paraíba que atenderam aos critérios: 1) Dispor do material de robótica da Fischertechnik ${ }^{\circledR}$; 2) Não dispor de espaço físico dedicado ou completamente organizado para aulas com robótica; 3) Não utilizar os materiais de robótica no ensino; 4) Apresentar interesse em operacionalizar o ensino com robótica.

A escola A selecionada oferta o EM Integral com o curso técnico em informática, enquanto a escola B está inserida no programa Escola Cidadã Integral com EM regular. Após a seleção, foram analisadas as condições dos laboratórios das instituições. A escola A possuía um espaço dedicado aos laboratórios de física, matemática e robótica, porém, a forma como os materiais de robótica estavam dispostos não facilitava seu uso. A escola B não possuía um espaço dedicado às aulas com robótica, os materiais estavam depositados em pequenas salas em condições que não favorecem seu uso por professores e alunos.

Considerando as condições das escolas, suas necessidades básicas e educacionais para o uso da robótica, foram estabelecidas regras para a organização dos laboratórios, a saber: 1) A sala deve comportar até 40 alunos; 2) A organização das mesas devem favorecer o trabalho em equipe; 3) O material didático impresso devem ser organizados por tipos em lugares acessíveis; 4) Os materiais dos kits de robótica devem ser arranjados por tipos em lugares acessíveis; 5) Os materiais (eletrônicos) dos kits de robótica devem ser contabilizados, e estarem dispostos em lugares acessíveis. É aconselhável conferência periódicas desses materiais pelo professor ou responsável devido sua importância e dificuldade de substituição. Em seguida, foi realizada a organização dos materiais das escolas, além da viabilização do espaço físico do laboratório da escola B. 
VII Congresso Brasileiro de Informática na Educação (CBIE 2018)

Anais dos Workshops do VII Congresso Brasileiro de Informática na Educação (WCBIE 2018)

\subsection{Atividades com Robótica}

As atividades foram organizadas no Construcionismo de [Papert 1980] em duas etapas. A primeira etapa contemplou a criação de soluções robóticas envolvendo conteúdos como, robô autônomo e inteligente; sustentabilidade; movimento; velocidade; tempo; escrita e leitura; entre outros. Foram aplicadas durante o segundo bimestre escolar com duas atividades que se desdobraram em quatro aulas, organizadas em três passos: 1) Contextualização: Apresentação da problemática envolvendo os alunos em discussões em busca de conhecimentos prévios; 2) Aprofundamento: Ligações dos conhecimentos consolidados com conceitos das ciências relacionados à temática da atividade; 3) Mão na massa: Construção livre de artefatos robóticos relacionados aos conceitos discutidos.

A segunda etapa focou na robótica fundamentada em computação e envolveu conceitos de algoritmo, raciocínio lógico e matemático, programação, entre outros. Foram aplicadas durante o terceiro bimestre escolar durante oito aulas organizadas em dois passos: 1) Teórica: Ensino e discussão de conceitos técnicos de robótica e computação; 2) Mão na massa: Partindo de uma situação problema, manipulação dos materiais de robótica para aplicação dos conceitos teóricos. As atividades e o programa de curso das atividades estão disponíveis para consulta no link: https://goo.gl/5PTWz9.

\subsection{Participantes}

Fizeram parte do estudo gestores e alunos da $1^{\text {a }}$ Série do EM de duas escolas estaduais da Paraíba. Ao todo, foram 41 alunos, sendo 20 da escola A (EM integral técnico) e 21 da escola B (EM integral regular). A amostra representa cerca de 86,9\% dos alunos participantes das aulas na escola A e 95,4\% na escola B. Foi considerada ainda, a opinião de 1 gestor da escola A e outro da B. As atividades foram realizadas durante o horário de aula, portanto, os dados coletados dizem respeito apenas aos alunos que estavam presentes nos dias da aplicação das aulas e dos questionários (pré-teste e pós-teste).

Segundo o censo escola a escola A possui 701 alunos matriculados no EM em 2018, enquanto que a escola B possui 1081. Na escola A, a turma selecionada representa $50 \%$ das 2 turmas de $1^{\text {a }}$ Série do EM ativas no ano letivo de 2018, enquanto que a turma da escola B, representa $16,7 \%$ das 6 turmas de $1^{\text {a }}$ Série do EM ativas no mesmo ano. A escolha das turmas considerou critérios que minimizavam a diferença entre as turmas e que favorecessem melhores condições para a realização das atividades planejadas, a saber: faixa etária, índice de reprovação, quantitativo de alunos e conhecimentos prévios em lógica de programação.

\subsection{Coleta e Análise dos Dados}

Os dados dos gestores foram coletados por meio de uma entrevista estruturada com perguntas envolvendo a chegada dos materiais de robótica, motivos e dificuldades que resultaram na não utilização da robótica, e por fim, como o trabalho realizado na escola é visto por eles. Os dados dos alunos foram coletados de duas formas: 1) Survey com questionamentos em torno do estímulo em aprender nas aulas, organização das aulas e estrutura física dos laboratórios; 2) Pré-teste e pós-teste (iguais) relacionados à lógica de programação, antes e após as intervenções/atividades descritas anteriormente. Os testes foram constituídos de seis questões, no formato de pseudocódigo criados por especialistas em computação, envolvendo estruturas de repetição e decisão, funções, contadores, 
vetores, além de variáveis e expressões aritméticas, os quais foram tabelados e analisados estatisticamente utilizando testes de proporção amparado pelo software $R$ versão 3.0.3. Os questionários e testes aplicados com os alunos, assim como o roteiro das entrevistas com os gestores estão disponíveis no link: https://goo.gl/5PTWz9.

O desempenho dos alunos nos testes foi analisado para avaliar se há indícios de que atividades com robótica podem auxiliar no desenvolvimento do PC. Para calcular e analisar o efeito das atividades no desempenho dos alunos, foi considerado o teste de proporção, observando a diferença $(\Delta)$ entre o desempenho no pré-teste (T1) e o desempenho no pós-teste (T2). Assim, quando: $\Delta>0$, o aluno apresentou melhora no desempenho do pós-teste; $\Delta=0$, o aluno apresentou melhora insignificante no desempenho do pós-teste; $\Delta<0$; o aluno apresentou piora no desempenho do pós-teste.

\section{Resultados e Discussões}

Este tópico, apresenta os resultados, discussões e ameaças à validade do presente estudo.

\subsection{Q1: Como a inserção da robótica impacta escolas de EB?}

Para responder a Q1 foram consideradas informações das entrevistas com os gestores. De acordo com os entrevistados, todo recurso educacional ajuda o ensino e fortalece a escola. Entretanto, a robótica se destaca por envolver o aluno em uma aprendizagem lúdica.

O gestor da escola A considera que o fato "dos alunos poderem participar, terem aulas, conhecerem os materiais, e verem a importância" é o principal impacto do trabalho, pois facilitar o aprendizado é o dever da escola. Ele aponta que a ampliação das aulas para toda a escola seria uma conquista, "pois muitos alunos veem o laboratório, mas não enxergam a possibilidade de aprendizagem", visão que o gestor afirma ter sido absorvida pelos participantes. Entretanto, o gestor destaca que a utilização da robótica pode impactar negativamente, uma vez que embora haja suporte inicial do governo com formações e orientações sobre a organização da sala, a escassez de profissionais habilitados na escola dificulta o uso do recurso, gerando o sentimento de frustração na equipe gestora.

O gestor da escola B afirma que o trabalho agiu nos alunos motivacionalmente, asseverando que "hoje os alunos estão motivados e nos cobram a participação em eventos de robótica". Além de motivar os alunos que estão participando, o trabalho impactou outros alunos que passaram a acreditar na possibilidade de terem contato com robótica. Hoje, a robótica é utilizada na escola B por outros professores, ou seja, os professores foram impactados. Porém, o gestor sinaliza que para a escola utilizar o material é complicado. O suporte pedagógico e técnico do governo não atende todas as necessidades para que a escola tenha autonomia em utilizar o material, tal como um livro didático. Com isso, é observado o desencanto em alunos que voluntariamente se propõem a participarem de eventos, mas que não atingem os objetivos por falta de orientação. Os professores, por diversas razões, não demonstram interesse em utilizar o material, restringindo as possibilidades educacionais da escola. Segundo o gestor da escola B, hoje os materiais são utilizados pelos professores da escola devido ao apoio dado pelo projeto aqui descrito.

Dessa forma, é observado que um trabalho metodologicamente elaborado é capaz de impactar positivamente a dinâmica escolar, sobretudo no fator motivacional que envolve alunos e professores da EB. Contudo, é importante que as colocações negativas dos gestores sejam consideradas para que as adversidades no uso da robótica sejam atenuadas. 
a)

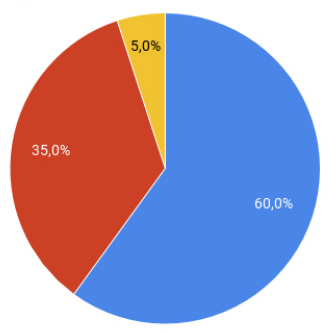

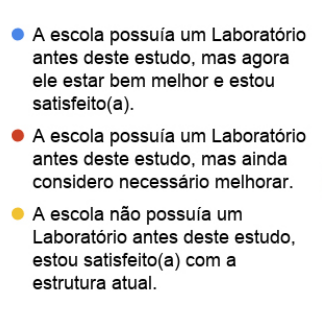

b)

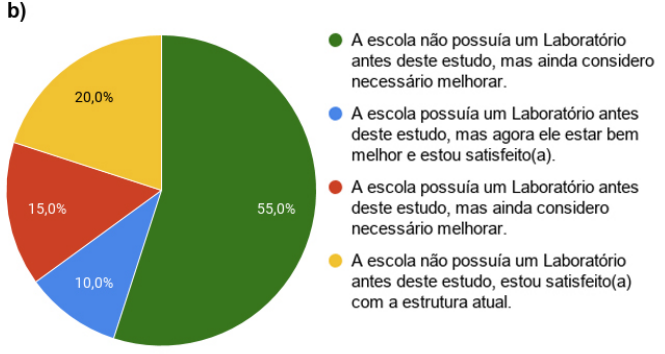

Figura 1. Opinião dos alunos sobre os laboratórios: a) Escola A; b) Escola B.

\subsection{Q2: Como a estrutura física e organizacional dos laboratórios influenciam na efetivação do ensino com robótica em escolas de EB?}

Para responder a Q2 foram considerados os dados das entrevistas com os gestores e o survey com os alunos. Os gestores acreditam que não é possível utilizar a robótica sem o espaço adequado e profissionais habilitados. Na escola A embora houvesse um espaço para a robótica, a forma como os materiais estavam armazenados não favorecia aos professores o uso. Hoje, mesmo sem professores experientes em robótica, o gestor acredita que o laboratório pode ser usado pelos próprios professores da escola A.

$\mathrm{Na}$ Escola B, onde a intervenção desta pesquisa atuou fortemente na organização, o gestor destaca que a existência de um espaço físico para a robótica é o princípio para que a escola utilize a ferramenta. "Nosso material saiu de dentro de armários para cima de bancadas, onde alunos e professores podem tocar e trabalhar juntos" e, de forma gratificante, é visto a expansão gradativa do uso da robótica pelos professores da escola.

Dos 41 alunos das duas escolas, 95,1\% (39) não tiveram contato com robótica anteriormente, enquanto 4,9\% (2) tiveram contato, ou seja, há uma consonância entre as colocações dos gestores e as respostas dos alunos no contexto do uso da robótica. De acordo com a Figura 1 ao serem questionados sobre a estrutura física dos laboratórios, $60 \%$ (12) dos alunos da escola A e 55\% (11) dos alunos da escola B demonstraram satisfação com a forma em que os laboratórios foram organizados.

Sobre a motivação em aprender com as aulas de robótica, 85\% (17) dos alunos da escola A e 90\% (18) da escola B indicaram serem motivados. Esses fatores mostram que o trabalho de organizacional foi observado pelos alunos, e a ações que a nova estrutura favoreceu, podem ter motivado os mesmos a aprenderem. Essas evidências se alinham ao posicionamento dos gestores no tocante à aprendizagem e motivação como principais fatores de impacto que o trabalho realizado causou nas escolas.

\subsection{Q3: Como ensino com robótica influencia as habilidades do Pensamento Computacional de alunos da EB?}

Para responder a Q3 foi considerado o desempenho dos alunos em lógica de programação nos testes através de seis questões, todas com opção de resposta "Não sei", buscando investigar a motivação em responderem, assim como os conceitos conhecidos por eles.

Segundo a Figura 2, em ambas as escolas, o número de tentativas de respostas no pós-teste, ou seja, a quantidade de respostas diferente de "Não sei" que cada questão recebeu, foi visivelmente maior quando comparada à quantidade das questões do pré- 
a)

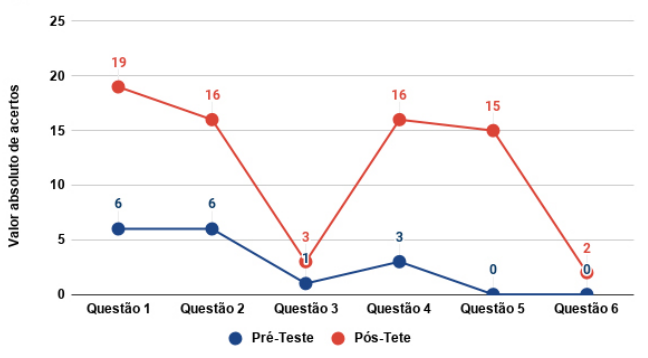

b)

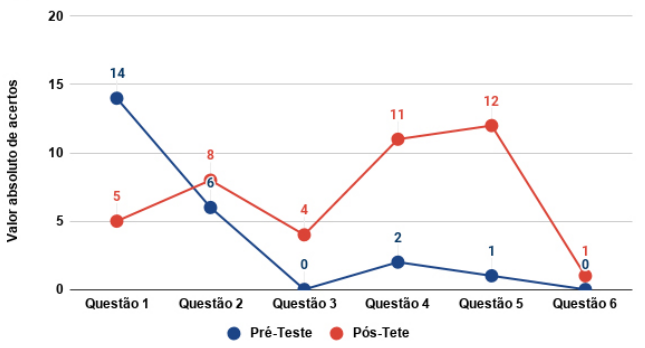

Figura 2. Quantidade de respostas por questão: a) Escola A; b) Escola B.

teste. Esses dados indicam que os alunos, antes das atividades com robótica possuíam pouca motivação em responder questões com conceitos de lógica de programação ou até mesmo desconhecimento do assunto. Após as atividades, considerando o aumento na tentativa de respostas, os alunos demonstraram maior motivação ou conhecimento sobre o assunto, o que impulsionou a tentativa de responder os questionamentos relacionadas à computação. É provável que a motivação destacada seja consequência do aprendizado de conceitos de computação durante as atividades realizada, o que pode impactar diretamente as habilidade do PC. É entendido por motivação, o fato do aluno se envolver com as questões na tentativa de respondê-las, mesmo que não seja a resposta correta, pois a partir da observação no decorrer da aplicação do pré-teste, foi percebido que muitos alunos não se empenharam em tentar responder fixando na resposta "Não sei".

Os dados da Figura 2 indicam as tentativas de respostas e não a corretude das mesmas, sendo necessário observar o desempenho. Este estudo, mensurou o desempenho a partir da quantidade de questões acertadas pelos alunos. Assim, para verificar a melhora do desempenho em lógica de programação dos participantes, consideramos o teste de proporção, observando o $\Delta$ entre o desempenho no pré-teste e pós-teste. Na escola $\mathrm{A}$ constatamos que $80 \%$ (16) dos alunos atingiram melhora no resultado final, mesmo que em alguns casos não seja o resultado ideal ( $p$-value $<0.003644$, nível de significância de 0.05). Contudo, na escola B apenas $38 \%$ (8) dos alunos atingiram melhora no resultado final, mesmo que em alguns casos não seja o resultado ideal ( $p$-value $<0.86233$, nível de significância de 0.05 ). Nesse sentido, foi obtido um $p$-value $>0.05$, logo pelo teste de proporção, menos de $50 \%$ dos alunos alcançam resultados minimamente aceitáveis.

Embora a quantidade de alunos que melhoraram seu desempenho na escola B seja equivalente a menos da metade dos alunos, verificamos que 57\% (12) dos alunos apresentaram melhora irrelevante (Tabela 1). Esses dados aliados ao índice motivacional dos alunos da escola B, indicam que um trabalho mais longitudinal com os alunos, pode converter (parcialmente ou completamente) esses desempenhos em sucesso. Essa realidade também se estende à escola A. É importante ressaltar que em ambas as escolas, 95,1\% dos alunos participantes tiveram o primeiro contato com robótica.

Tabela 1. Teste de proporção no desempenho em conceitos de computação

\begin{tabular}{|c|c|c|c|c|c|}
\hline Escola & Sucesso & Irrelevante & Fracasso & $\boldsymbol{p}$-value & Significância \\
\cline { 1 - 5 } A & 16 & 3 & 1 & $<0.003644$ & 0.05 \\
\hline B & 8 & 12 & 1 & $<0.86233$ & \\
\hline
\end{tabular}


De modo geral, atividades com robótica considerando fatores físicos, estruturais e metodológicos, aliados aos conceitos de computação, podem motivar alunos a aprenderem e possivelmente estimular o PC na EB, como evidenciado pela melhoria no desempenho dos alunos em questões de lógica de programação. É possível que a inserção da robótica na EB seja capaz de, ao longo do tempo, melhorar os efeitos no desempenho acadêmico dos alunos em conceitos de lógica de programação, refletindo nas habilidades do PC e consequentemente no aprendizado das demais disciplinas curriculares.

\subsection{Ameaças à Validade}

Este estudo apresenta ameaça de construção relacionada ao planejamento das atividades, pois os cenários distintos que foram aplicadas podem requerer abordagens também distintas. Apresenta ameaças internas, pois muitos alunos não estavam presentes durante a aplicação dos testes ou em mais de $70 \%$ das aulas. Os alunos que responderam os testes e o survey podem não ter entendido, não ter dado atenção às perguntas ou não ter respondido com franqueza. Apresenta ameaças de conclusão, pois o tamanho da amostra resulta em baixo poder estatístico, além disso, os alunos da escola A ao terem contato com outras disciplinas de informática podem estar mais expostos a estímulos do PC. Por fim, apresenta ameaças externas por considerar amostras de alunos da $1^{\text {a }}$ Série do EM de duas escolas estaduais da Paraíba, não sendo possível estender os resultados para outras populações de alunos.

\section{Considerações Finais}

Este estudo observou os impactados que o trabalho com robótica causou em escolas de EB do estado da Paraíba. Foi realizado um trabalho dividido em três fases: 1) Seleção e organização de laboratório de robótica em escolas estaduais de EB; 2) Construção e aplicação de atividades com robótica para alunos da $1^{\text {a }}$ Série do EM; 3) Exploração dos efeitos que o trabalho com robótica causou nos alunos participantes.

A investigação indicou que um trabalho com robótica metodologicamente elaborado é capaz de impactar positivamente a formação e motivação de alunos, assim como de professores da EB que passam a utilizar a ferramenta no ensino quando condições mínimas são disponibilizadas. Foi evidenciado que a organização física dos laboratórios de robótica é considerada por gestores e alunos como importante, e que aulas de robótica realizadas em estrutura física organizada podem motivar o aprendizado. Por fim, verificamos que $80 \%$ e $38 \%$ dos alunos das escolas A e B, respectivamente, apresentaram melhora no desempenho dos testes envolvendo conceitos de lógica de programação. Os dados indicam que o PC de alunos da EB pode ser melhorado quando a robótica é utilizada no ensino considerando fatores físicos, estruturais e metodológicos.

As questões apresentadas são evidências preliminares de uma pesquisa de mestrado em CC que busca estimular o PC na EB através da RE. Esperamos que os resultados auxiliem a investigação dos efeitos que a RE causa no PC, e consequentemente, no aprendizado de alunos da EB nas disciplinas curriculares. Esperamos ainda, auxiliar escolas e professores de EB na utilização da RE, abordando reflexões sobre às condições básicas dos laboratórios, assim como práticas que orientem o uso dessa ferramenta no ensino.

Pretendemos utilizar instrumentos que explorem melhor as habilidades do PC, assim como, aplicar recursos estatísticos mais poderosos para analisar profundamente o 
VII Congresso Brasileiro de Informática na Educação (CBIE 2018)

Anais dos Workshops do VII Congresso Brasileiro de Informática na Educação (WCBIE 2018)

desenvolvimento das habilidades relacionadas ao PC no decorrer das atividades com RE na EB e entender os fatores envolvendo as duas escolas apresentadas neste trabalho.

\section{Referências}

Araujo, A. L., Andrade, W., and Guerrero, D. (2016). Um mapeamento sistemático sobre a avaliaçao do pensamento computacional no brasil. In Anais dos Workshops do CBIE.

Barr, V. and Stephenson, C. (2011). Bringing computational thinking to k-12: What is involved and what is the role of the computer science education community? ACM.

Cohen, L., Manion, L., and Morrison, K. (2002). Research methods in education. Routledge.

Computer Science Teachers Association, C. (2011). Computational thinking in k-12 education. Disponível em: https://goo.gl/qcqJia.

Costa, E. J. F., Sampaio, L., and Guerrero, D. (2016). Pensamento computacional na educação básica: Uma análise da relação de questões de matemática com as competências do pensamento computacional. In Anais dos Workshops do CBIE.

Dicionário Interativo da Educação Brasileira, D. (2015). Definição de robótica educacional. www.educabrasil.com.br.

França, R. d., Silva, W. d., and Amaral, H. d. (2012). Ensino de ciência da computaçao na educaçao básica: Experiências, desafios e possibilidades. In Anais dos Workshop $C S B C$.

Gerber, L. C., Calasanz-Kaiser, A., Hyman, L., Voitiuk, K., Patil, U., and Riedel-Kruse, I. H. (2017). Liquid-handling lego robots and experiments for stem education and research. PLoS biology, 15(3):e2001413.

Halfpap, D. M. et al. (2005). Um modelo de consciência para aplicação em artefatos inteligentes.

Matos, E. d. S. (2013). Integração curricular por meio da prática de ensino interdisciplinar em ihc. In WEIHC, pages 25-30.

Papert, S. (1980). Logo: Computadores e educação. são paulo: Brasiliense. A Máquina das Crianças. Porto Alegre-RS: Artmed.

Rodrigues, R. d. S., Andrade, W., Guerrero, D., and Sampaio, L. (2015). Análise dos efeitos do pensamento computacional nas habilidades de estudantes no ensino básico: um estudo sob a perspectiva da programação de computadores. In SBIE / CBIE.

Santos, O. L. d., Cury, D., Rafalski, J., and Silveira, P. D. N. (2016). An iot computational robotics learning laboratory in vila velha, espírito santo. In LACLO.

Souza, I. M. L. d., da Silva Rodrigues, R., and Andrade, W. (2016). Explorando robótica com pensamento computacional no ensino médio: Um estudo sobre seus efeitos na educação. In SBIE / CBIE), volume 27.

Strnad, B. (2017). Programming lego mindstorms for first lego league robot game and technical interview. In 40th International Convention on. IEEE.

Wing, J. M. (2006). Computational thinking. Communications of the ACM, 49(3):33-35. 